\title{
Spectral Reflectance Processing via Local Wavelength-Direction Correlations
}

\section{Xu, Guanglang}

2020-06

Xu , G , Gritsevich , M , Peltoniemi , J , Penttilä , A, Ihalainen, O , Wilkman , O \& Muinonen , K 2020 , ' Spectral Reflectance Processing via Local Wavelength-Direction Correlations ' , IEEE Geoscience and Remote Sensing Letters , vol. 17 , no. 6 , pp. 948-952 . https://doi.org/10.1109/LGRS.2019.29

http://hdl.handle.net/10138/315663

https://doi.org/10.1109/LGRS.2019.2939228

acceptedVersion

Downloaded from Helda, University of Helsinki institutional repository.

This is an electronic reprint of the original article.

This reprint may differ from the original in pagination and typographic detail.

Please cite the original version. 


\title{
Spectral Reflectance Processing via Local Wavelength-Direction Correlations
}

\author{
Guanglang $\mathrm{Xu}^{\oplus}$, Maria Gritsevich, Jouni Peltoniemi, Antti Penttilä, Olli Wilkman, Olli Ihalainen, \\ and Karri Muinonen
}

\begin{abstract}
The spectral bidirectional reflectance distribution function (BRDF) maps incident radiation of a surface to its outgoing counterpart at different wavelengths. This function plays a fundamental role in characterizing the various types of earth surfaces. Due to its high dimensionality, the measurements, analysis, and simulation of spectral BRDF are challenging. In this letter, we introduce a new method for processing spectral reflectance using the so-called data-adjacency, i.e., the correlation between adjacent wavelengths and viewing directions. The results show that the benefits of efficient representation, noise reduction, and analysis capability can all be integrated to the data.
\end{abstract}

Index Terms-Remote sensing, spectroradiometers, wavelet transforms.

\section{INTRODUCTION}

$\mathbf{T}$ HE electromagnetic waves detected by the satellite sensors are, in general, dependent on viewing and illumination directions at different wavelengths. Such information about the earth surface is encoded in the spectral bidirectional reflectance distribution functions (BRDFs). The importance of spectral BRDFs for remote sensing is fundamental [1]. Over decades, the remote sensing satellites have been built in order to obtain the higher resolution in wavelength, enhancing the angular detection capabilities, e.g., the hyperspectral and multi-angular satellites. Meanwhile, the earth radiation estimation relies on modeling the spectral BRDFs of various atmospheric, oceanic, and land components [2]-[4] via radiative transfer simulations. From a computational perspective, accurate spectral BRDF models are also needed as the boundary conditions in radiative transfer.

Currently, many of the spectral BRDF models are either theoretical or empirical [5]-[7], few are based on field and

Manuscript received June 5, 2019; revised August 13, 2019; accepted August 31, 2019. This work was supported by the Academy of Finland Consortium Project Albedo under Project 298137 and Project 298139. (Corresponding author: Guanglang Xu.)

G. $\mathrm{Xu}, \mathrm{A}$. Penttilä, and $\mathrm{O}$. Ihalainen are with the Department of Physics, University of Helsinki, 00100 Helsinki, Finland (e-mail: guanglang.xu@helsinki.fi).

M. Gritsevich is with the Department of Physics, University of Helsinki, 00100 Helsinki, Finland, also with the Institute of Physics and Technology, Ural Federal University, 620002 Yekaterinburg, Russia, and also with the Finnish Geospatial Research Institute FGI, National Land Survey of Finland, 00520 Helsinki, Finland.

J. Peltoniemi and O. Wilkman are with the Finnish Geospatial Research Institute FGI, National Land Survey of Finland, 00520 Helsinki, Finland.

$\mathrm{K}$. Muinonen is with the Department of Physics, University of Helsinki, 00100 Helsinki, Finland, and also with the Finnish Geospatial Research Institute FGI, National Land Survey of Finland, 00520 Helsinki, Finland.

Color versions of one or more of the figures in this letter are available online at http://ieeexplore.ieee.org.

Digital Object Identifier 10.1109/LGRS.2019.2939228 laboratory measurements. There are a few reasons for this. First, the measurements are simply too costly for many of the purposes compared to the theoretical models. Second, there is a lack of systematic or efficient ways to convert the measured data set (usually incomplete) to applicable models [8]. In this letter, we present a new approach aiming to overcome some of the inefficiency in dealing with the spectral reflectance data, integrating the benefits of efficient representation, and analysis capability as well as noise reduction.

\section{FOLD-AND-UNFOLD SCHEME}

By definition, the spectral BRDFs cannot be directly measured, the term "BRDF data" refers to derived values from a measurable quantity called bidirectional reflectance factor (BRF) [9]. Considering its most general case, the spectral BRDF for a particular surface is dependent on the incident and outgoing directions, wavelength, and polarization state of the electromagnetic waves. Making full measurements and storing them in high resolution would be incredibly costly [10]. In this section, the so-called fold-and-unfold scheme is presented for dealing with the spectral BRDF data. Note that due to its fundamental role in scientific and engineering applications [1], [11], the method presented here could potentially be extended to other contexts, in addition to its applications in remote sensing, radiative transfer, and earth albedo estimation. The data applied in this letter are from a comprehensive spectral BRF library built by the Finnish Geospatial Research Institute (FGI) over many years. This data set provides various earth's surface reflectance properties, including snow, vegetation, sand, and so on. A short description of our data source is attached in Appendix D.

\section{A. Wavelet-Based Representation}

The BRDFs often show peaked values at certain directions, which could be caused by specular reflections or other single- or multiple-scattering mechanisms. This makes some of the conventional analytical tools such as spherical harmonics less useful. It is a common practice of combining the Dirac delta function and spherical harmonics to represent BRDF. Nevertheless, this way of representation is not very natural [12]-[14]. The spherical harmonics provide global representation of a function and, therefore, local irregularity could lead to global distortion. In addition, the continuity between the sharp and diffuse components cannot be properly handled by simply combining the Dirac delta function and spherical harmonics. In this letter, we turn to more practical tools known as spherical wavelets [15]-[17]. The spherical wavelets 


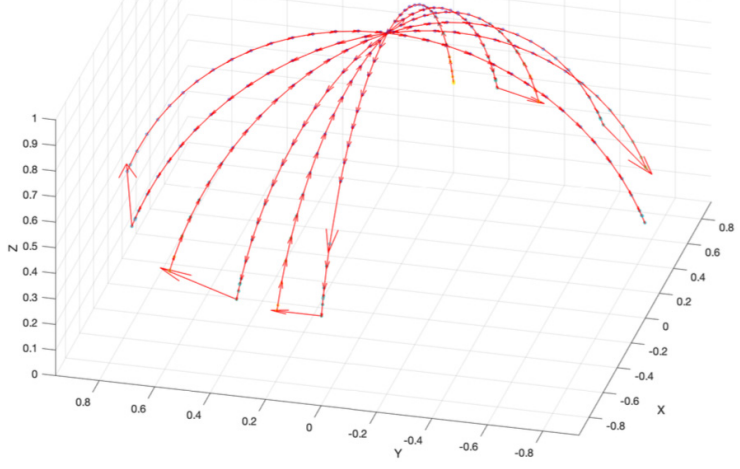

Fig. 1. Parameterizing the reflectance via data adjacency. The vectors indicate the moving directions of the detector on the hemisphere.

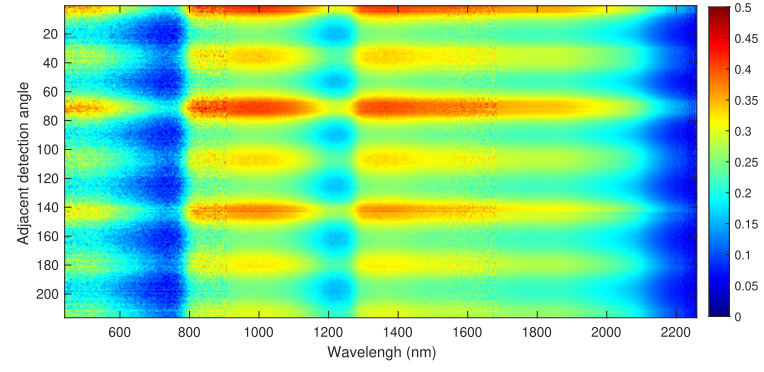

Fig. 2. Adjacency parameterization of a sand surface spectral BRDF sample from the FGI BRDF library. The color maps to the reflectance data.

provide local support on the surface of a sphere, which means that local irregularity will not lead to global distortion [18]. Moreover, the wavelet-based representation is equipped with multiresolution analysis (MRA), which is useful for analysis and representation of the data. More technical details about the spherical wavelets and and their implementations are described in [15], [16], and [18]-[20]. A short introduction to this technique is also attached in the appendices.

\section{B. Parameterization via Data Adjacency}

The basic idea of representing the spectral BRDFs more efficiently is to take advantage of the correlation between all neighboring quantities, in both wavelength and direction. Accordingly, we propose a nonmetric-parameterization of the spectral BRDF. Specifically, the direction hemisphere is parameterized to a line of adjacent directions in accordance with measurement, as displayed in Fig. 1. Mathematically, this is equivalent to mapping a spherical surface to a line, i.e., $S^{2} \rightarrow R$. Obviously, the metric property is no longer preserved. The resulting parameterization of the spectral data become $S^{2} \times R \rightarrow R \times R$ for a fixed incident direction (a full representation is $S^{2} \times S^{2} \times R$, including all possible incident directions). The adjacent detection angles are composed of the directions in neighboring meridian planes. They are in line with the detector's movement; hence, they have local spatial correlations, as displayed in Fig. 2. They are in line with the detector's movement; hence, they have local spatial correlations.
In order to fully utilize the correlations between wavelength and direction, the data are then mapped to a spherical surface, i.e., $S^{2} \times R \rightarrow R \times R \rightarrow S^{2}$. This mapping could be just a linear interpolation of azimuthal and zenith angles. It should be noted that this mapping is not strictly required in order to obtain the benefits of denoising and efficient representation. However, there are some extra benefits brought up by this spherical mapping. First, the compression efficiency is higher after mapping the data to a spherical surface. This is because there is no boundary on the spherical surface and the local correlations will be optimally explored. Second, the BRDF data have naturally a spherical structure, and they are inherently connected with spherical coordinates. Third, direct computing and analysis of the data could be more conveniently achieved by representing the data on the spherical surface.

\section{Multi-Resolution Processing}

This adjacency-based parameterization ensures that each data point on the surface has certain degree of correlation with the adjacent points, and this provides a playground for spherical wavelet multi-resolution analysis [21]. Now, the data can be seen as a function on the sphere $f$. The next step is to perform spherical wavelet transform in order to decompose $f$

$$
f=\sum_{k \in K^{0}} c_{k}^{J_{0}} \tilde{\phi}_{k}^{J_{0}}+\sum_{j=J_{0}}^{J} \sum_{m \in M^{j}} d_{m}^{j} \tilde{\psi}_{m}^{j}
$$

where $j$ is the index of the decomposition level, $J_{0}$ refers to the coarsest level, $J$ refers to the finest level, $\tilde{\phi}_{k}^{J_{0}}$ is the dual scaling function at the coarsest level, $\tilde{\psi}_{m}^{j}$ is the dual wavelet function at level $j, c_{k}^{J_{0}}$ are the coefficients giving the coarsest representation of $f$, and $d_{m}^{j}$ are the wavelet coefficients of each level. The above expression is, indeed, a recovery formula of $f$, feeding the coefficients to construct the original data. In order to obtain the coefficients for those basis functions, one must perform the inner products with the scaling functions and wavelet functions

$$
c_{k}^{J_{0}}=\left\langle f, \phi_{k}^{J_{0}}\right\rangle, \quad d_{m}^{j}=\left\langle f, \psi_{m}^{j}\right\rangle
$$

where the $\phi_{k}^{J_{0}}$ is the primal scaling function, and $\psi_{m}^{j}$ is the primal wavelet function. Equations (1) and (2) correspond to two processes, namely, the forward wavelet transform and inverse wavelet transform. Many of the benefits can be achieved by performing the forward and inverse wavelet transforms, including obtaining multi-resolution spectral data, noise reduction, and efficient representation.

\section{Local Wavelength-Direction Correlations}

At first glance, the parameterization seems to obscure the angular distance between two data points. In fact, the parameterization trades the metric properties with adjacency; as such, the local correlations of the spectral BRDF can be optimally explored in order to obtain efficient representation. There is no need to take in the real metric information for wavelet transform, while the local correlations are crucial for the performance of wavelet transform. As for the real angular distances and coordinates, they can always be regained after the forward and backward wavelet transforms. The wavelet 


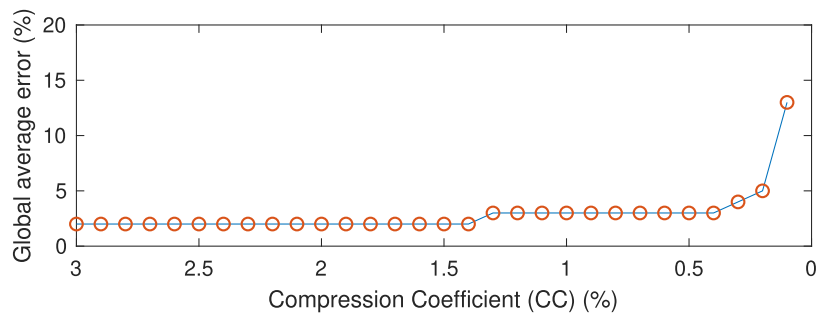

Fig. 3. Global average error as a function of $\mathrm{CC}$, defined as the ratio of number of surviving coefficients to the number of original data points for the whole spectral BRDF data.

transforms are built on different levels of triangulated geodesic grids on the sphere, and they are essentially combinatorial. In this letter, we construct the multi-scale bases using the Lifting Scheme [19], [20]. With the scheme, one could start with a trivial base such as the Lazy-wavelet [15] and incrementally obtain more performant bases. It includes three basic processes, namely, the split, predict, update, and their corresponding reverses [15].

\section{RESUlTS AND DisCUSSION}

\section{A. Effectiveness of Representation}

As mentioned above, the basic idea is to map the spectral BRDF data to a spherical surface to explore the local correlations between wavelengths and directions. For most of the points on the surface, the data tends to vary smoothly thanks to the adjacency-based parameterization. We then use the Lifting Scheme to perform the fast wavelet transform to obtain the scaling function coefficients and wavelet coefficients. The next step is to perform thresholding to the obtained coefficients, i.e., keeping the large coefficients untouched and setting all small ones to zeros. To construct the spectral data, we perform the inverse wavelet transform using the thresholded coefficients. We define the compression coefficient (CC) as the ratio of the number of nonzero coefficients to the number of original data points. Therefore, a smaller CC value corresponds to a higher efficiency of representation. As such, the whole foldand-unfold scheme can be described as follows:

$$
S^{2} \times R \underset{\text { metric }}{\stackrel{\text { adjacency }}{\rightleftharpoons}} R \times R \rightleftharpoons S^{2} \underset{\text { BWT }}{\stackrel{\text { FWT }}{\rightleftharpoons}} S^{2}
$$

where FWT and BWT denote the forward and backward wavelet transforms, respectively, and the second operation $\left(R \times R \rightleftharpoons S^{2}\right.$ ) corresponds to the mixing of wavelength and spatial information. With this folding and unfolding strategies, a very small $\mathrm{CC}$ value can be obtained for the whole spectral BRDF data. Fig. 3 displays the global average error as a function of the $\mathrm{CC}$ value. As shown in the figure, the global average error does not grow much until the $\mathrm{CC}$ value reaches a very small number of $0.1 \%$. In contrast to the metric-preserving parameterization, there is no need to perform wavelet-transform for each wavelength, and this dramatically increases the effectiveness of representation.

\section{B. Noise Reduction and Detail Extraction}

It is well known to the experimentalist that the BRDF measurement often comes with severe noise issues [11], [22]. With the wavelet transforms, efficient representation of the
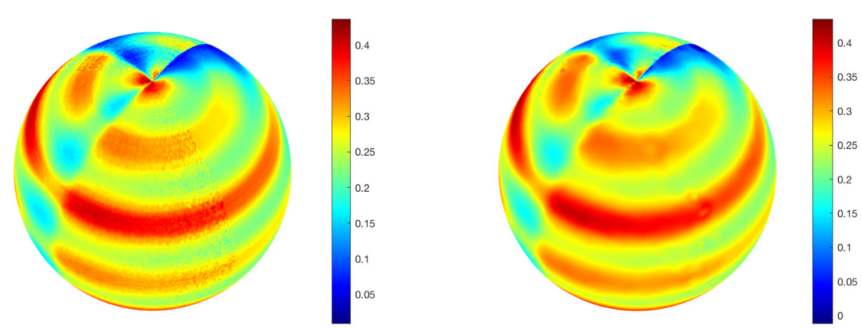

Fig. 4. (Left) Spherical representation of the spectral reflectance data. (Right) Noise reduction can be obtained by tuning the compression rate.

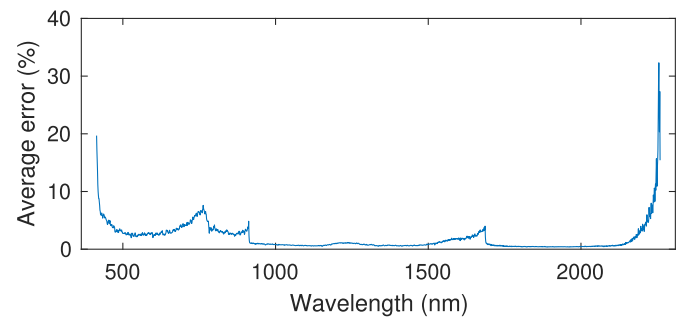

Fig. 5. Detail extraction across the spectrum.

data and noise reduction can be achieved at the same time. This is done by controlling the global error while reducing the number of nonzero coefficients. Fig. 4 displays the spherical representation of reflectance data (left panel) and its noise reduction (right panel) by controlling the global difference around $3 \%$.

As demonstrated in the appendix, the wavelet transform is a process of separating coarse representation and local details in the data. The details are represented by the wavelet coefficients. If one subtracts the smoothed version of the data from the original data, we will have the details of the data. We call this process the detail extraction. Fig. 5 displays the spectral difference distribution with a global average difference of $3 \%$. The detail extraction could be useful for detecting some artifacts in the data. For example, the small peak at wavelength around $1700 \mathrm{~nm}$ in Fig. 5 corresponds to a transition of high-level noise to low-level noise in the original data. In addition, as displayed in Fig. 5, the shortest and longest wavelengths tend to have the largest detail, and this is due to the adjacency-based parameterization. Because there are no neighboring wavelengths for the shortest and longest ones, the wavelet coefficients are much larger than those in the middle.

\section{Discussion}

Based on the results presented, there are some implications we would like to discuss here. First, in terms of representation, local "coordinates" are more effective than global "coordinates." This effectiveness, more specifically, is with respect to obtaining sparseness in the representation. As more and more sensors are being manufactured and used to collect data, obtaining sparseness and compressibility becomes more and more important. Second, multi-scale processing is a very natural way of processing the reflectance data. As demonstrated in this letter, by decomposing the signal into different scales and exploring the connections between two nested scales, we can obtain useful information such as noise level transition across the spectrum. 


\section{CONCLUSiON}

In this letter, we present a novel approach for spectral reflectance data processing based on the spherical wavelet technique. The results show that the method integrates the benefits of efficient representation, noise reduction, and analysis capability to the data. We shall point out the characteristics of the method more specifically. First, the reflectance is parameterized in such a way that we can take advantage of the correlations between neighboring wavelengths and directions at the cost of preserving the metric properties. Second, the parameterization is in line with the detector's movement, and therefore, it provides a path for real-time processing. The method is applied to the whole spectral data. There is no need to represent the BRDF for each wavelength; hence, the compressed data will not grow linearly with the number of wavelengths. This could be very useful for high-spectral resolution BRDF processing. Some future work is noted as follows.

1) The Fold-Expand Scheme: As mentioned beforehand, our current work is partly motivated by converting a comprehensive spectral BRDF library to applicable models. One direction is to develop a method to "expand" the data from the incomplete measurements in order to obtain a full $S^{2} \times S^{2} \times R$ spectral BRDF representation. We intended to apply the state-of-theart technique called compressive sensing [6], [23], [24], which is in close relation to data compression methods.

2) Direct Computing and Analysis on $S^{2}$ : The wavelength-direction correlations provide richer information about the spectral characteristics than noncorrelated ones. The scheme could be applied to spectral characteristics learning and analysis. With the spherical wavelet techniques, fast multi-resolution analysis over the spectrum can be obtained. In addition, we can incorporate real metric information for efficient computing on $S^{2}$.

\section{APPENDIX A \\ SPHERICAL WAVELETS AND MULTI-RESOLUTION \\ PROCESSING}

Spherical wavelets are constructed on the hierarchical geodesic grids on the sphere. These grids can be obtained by subdivision of the zero-level grids, the icosahedron. The multi-level discretization of a spherical surface naturally leads to the multi-scale representation and analysis of a function on the sphere. The bases for different scale representations are called the scaling functions $\phi_{k}^{j}$, and the spherical wavelets live in the complementing space between two nested scaling function spaces. Mathematically, this relation can be expressed as

$$
L_{2}=V_{0} \oplus \bigoplus_{j=0}^{\infty} W_{j}
$$

where $V_{0}$ denotes the coarsest scaling function space, and $W_{j}$ is the wavelet function space indexed by decomposition level. The function on the sphere $f$ can, therefore, be represented using the coarsest scaling functions and spherical wavelets at different complementing spaces [as shown in (1)]. For each space, the bases have their primal and dual counterparts, i.e., the primal/dual scaling functions and primal/dual wavelets. They are biorthogonal bases. Biorthogonality leads to lossless transform.

\section{APPENDIX B}

\section{REFINEMENT RELATION AND WAVELET CONSTRUCTION}

The central task of wavelet transform is to perform the following operations iteratively:

$$
\sum_{l \in K^{j+1}} c_{l}^{j+1} \tilde{\phi}_{l}^{j+1} \underset{\text { backward }}{\stackrel{\text { forward }}{\rightleftharpoons}} \sum_{k \in K^{j}} c_{k}^{j} \tilde{\phi}_{k}^{j}+\sum_{m \in M^{j}} d_{m}^{j} \tilde{\psi}_{m}^{j}
$$

where the index sets $K^{j}$ and $M^{j}$ satisfy

$$
K^{j+1} \underset{\text { Merge }}{\stackrel{\text { Split }}{\rightleftharpoons}} K^{j} \cup M^{j}
$$

where decomposition level $j$ decreases toward the forward direction and increases toward the backward direction. The wavelet coefficients will be spitted out via forward transform and absorbed via backward transform. We use the following refinement relations of scaling functions and the wavelet construction formula:

$$
\begin{aligned}
& \tilde{\phi}_{k}^{j}=\tilde{\phi}_{k}^{j+1}+\sum_{m \in N_{k}^{j}} h_{m, k}^{j} \tilde{\phi}_{m}^{j+1} \\
& \tilde{\psi}_{m}^{j}=\tilde{\phi}_{m}^{j+1}-\sum_{k \in A_{m}^{j}} s_{k, m}^{j} \tilde{\phi}_{k}^{j}
\end{aligned}
$$

where $N_{k}^{j}$ and $A_{m}^{j}$ are two sets of indices being locally correlated with $k$ and $m$, respectively, and they satisfy

$$
N_{k}^{j} \subset M^{j}, \quad A_{m}^{j} \subset K^{j} .
$$

The refinement relation for scaling functions together with the wavelet bases formula give their corresponding coefficient relations, that is,

$$
\begin{aligned}
c_{m}^{j+1} & =\sum_{k \in n_{m}^{j}} c_{k}^{j+1} h_{k, m}^{j}+d_{m}^{j} \\
c_{k}^{j+1} & =-\sum_{m \in a_{k}^{j}} d_{m}^{j} s_{m, k}^{j}+c_{k}^{j}
\end{aligned}
$$

where $n_{m}^{j}$ and $a_{k}^{j}$ are two sets of indices being locally correlated with $m$ and $k$, respectively, and they satisfy

$$
n_{m}^{j} \subset K^{j}, \quad a_{k}^{j} \subset M^{j} .
$$

\section{APPENDIX C \\ WAVElet Transform Via The LifTing SCHEME}

By using the coefficient transform relations described above, we will be able to perform the forward and backward wavelet coefficient transforms. It is fast in such a way that the computation could be in-place and only require minimum 
computational cost. The entire wavelet transform can now be represented as follows:

$$
\begin{aligned}
& \left\{c_{l}^{j+1}\right\} \underset{\text { merge }}{\stackrel{\text { split }}{\rightleftharpoons}}\left\{c_{k}^{j+1}\right\} \cup\left\{c_{m}^{j+1}\right\} \\
& \underset{\text { predict: } d_{m}^{j}=c_{m}^{j+1}-\sum_{k \in n_{m}^{j}} c_{k}^{j+1} h_{k, m}^{j}}{\rightleftharpoons}\left\{c_{k}^{j+1}\right\} \cup\left\{d_{m}^{j}\right\} \\
& \text { update: } c_{k}^{j}=\sum_{m \in a_{k}^{j}} d_{m}^{j} s_{m, k}^{j}+c_{k}^{j+1} \text {, } \\
& \text { reverse update: } c_{k}^{j+1}=-\sum_{m \in a_{k}^{j}} d_{m}^{j} s_{m, k}^{j}+c_{k}^{j} \text {, }
\end{aligned}
$$

The upper one is the forward transform, and the lower one is the backward transform. The computations can be iteratively carried out among many different levels. The filter $h_{k, m}^{j}$ corresponds to the design of a predictor, and $s_{m, k}^{j}$ corresponds to increasing the vanishing moments of wavelets.

\section{APPENDIX D \\ FGI BRF LIBRARY}

The examples of BRF measurements used in this letter are taken from a large BRF library measured by the Finnish Geodetic Institute Field Goniospectrometer (FIGIFIGO) from the FGI [25]-[30]. The primarily aim of FIGIFIGO is to provide multiangular spectropolarimetric measurements of various samples, including sand, snow, vegetation, etc., both under actual field conditions and in the laboratory using artificial illumination. The potential use of the data from the FIGIFIGO measurements is diverse; including their use as ground truth references for earth observation and remote sensing studies, testing and validation of theoretical scattering models, estimating climate change over time, measuring other ecological effects caused by changes in land cover, and more generally, to aid in the identification and analysis of both seasonal, and nonseasonal variations of targets of interest.

\section{ACKNOWLEDGMENT}

The authors would like to thank the anonymous reviewers for their constructive suggestions and comments.

\section{REFERENCES}

[1] J. Shell, Bidirectional Reflectance An Overview With Remote Sensing Applications Measurement Recommendations. Rochester, NY, USA: Rochester Institute of Technology, 2004.

[2] G. Xu, P. G. Stegmann, S. D. Brooks, and P. Yang, "Modeling the single and multiple scattering properties of soot-laden mineral dust aerosols," Opt. Express, vol. 25, no. 24, pp. A990-A1008, 2017.

[3] G. Xu, B. Sun, S. D. Brooks, P. Yang, G. W. Kattawar, and X. Zhang, "Modeling the inherent optical properties of aquatic particles using an irregular hexahedral ensemble," J. Quant. Spectrosc. Radiat. Transf., vol. 191, pp. 30-39, Apr. 2017

[4] J. Zenker et al., "Using depolarization to quantify ice nucleating particle concentrations: A new method," Atmos. Meas. Techn., vol. 10, no. 12, pp. 4639-4657, 2017.

[5] R. Montes and C. Ureña, "An overview of BRDF models," Univ. Grenada, Grenada, West Indies, Tech. Rep. LSI-2012, 2012, vol. 1.

[6] D. Guarnera, G. C. Guarnera, A. Ghosh, C. Denk, and M. Glencross, "BRDF representation and acquisition," Comput. Graph. Forum, vol. 35, no. 2, pp. 625-650, 2016.
[7] K. Muinonen and K. Lumme, "Disk-integrated brightness of a LommelSeeligerscattering ellipsoidal asteroid," Astron. Astrophys., vol. 584, p. A23, Sep. 2015.

[8] W. Matusik, H. Pfister, M. Brand, and L. McMillan, "Efficient isotropic BRDF measurement," in Proc. Eurograph. Symp. Rendering, 14th Eurographics Workshop Rendering, Jun. 2003, pp. 241-248.

[9] K. Z. Doctor, C. M. Bachmann, D. J. Gray, M. J. Montes, and R. A. Fusina, "Wavelength dependence of the bidirectional reflectance distribution function (BRDF) of beach sands," Appl. Opt., vol. 54, no. 31, pp. F243-F255, 2015.

[10] N. Seylan, S. Ergun, and A. Öztürk, "BRDF reconstruction using compressive sensing," in Proc. 21st Int. Conf. Comput. Graph., Vis. Comput. Vis., 2013, pp. 88-94.

[11] S. Rusinkiewicz. (1997). A Survey of BRDF Representation for Computer Graphics. [Online]. Available: http://www-graphics. stanford. edu/cs348c/surveypaper. html

[12] P. S. Shirley, "Physically based lighting calculations for computer graphics," Ph.D. dissertation, Univ. Illinois at Urbana-Champaign, Champaign, IL, USA, 1991

[13] L. Claustres, Y. Boucher, and M. Paulin, "Spectral BRDF modeling using wavelets," Proc. SPIE, vol. 4738, pp. 33-44, Mar. 2002.

[14] P. Lalonde and A. Fournier, "A wavelet representation of reflectance functions," IEEE Trans. Vis. Comput. Graphics, vol. 3, no. 4, pp. 329-336, Oct. 1997.

[15] P. Schröder and W. Sweldens, "Spherical wavelets: Efficiently representing functions on the sphere," in Proc. 22nd Annu. Conf. Comput. Graph. Interact. Techn., 1995, pp. 161-172.

[16] P. Schröder and W. Sweldens, "Spherical wavelets: Texture processing," in Rendering Techniques. Vienna, Austria: Springer, 1995, pp. 252-263.

[17] W. Sweldens, "Wavelets and the lifting scheme: A 5 minute tour," ZAMM-Zeitschrift Fur Angewandte Math. und Mech., vol. 76, no. 2, pp. 41-44, 1996.

[18] J.-P. Antoine and D. Roşca, "The wavelet transform on the two-sphere and related manifolds: A review," Proc. SPIE, vol. 7000, Apr. 2008, Art. no. 70000B.

[19] W. Sweldens, "The lifting scheme: A construction of second generation wavelets," SIAM J. Math. Anal., vol. 29, no. 2, pp. 511-546, 1998.

[20] W. Sweldens, "The lifting scheme: A custom-design construction of biorthogonal wavelets," Appl. Comput. Harmon. Anal., vol. 3, no. 2, pp. 186-200, Apr. 1996.

[21] S. Mallat, A Wavelet Tour of Signal Processing. Amsterdam, The Netherlands: Elsevier, 1999.

[22] T. A. Leonard and P. Rudolph, "BRDF round robin test of ASTM E1392," Proc. SPIE, vol. 1995, pp. 285-294. Dec. 1993.

[23] D. L. Donoho, "Compressed sensing," IEEE Trans. Inf. Theory, vol. 52, no. 4, pp. 1289-1306, Apr. 2006.

[24] E. J. Candès, J. Romberg, and T. Tao, "Robust uncertainty principles: Exact signal reconstruction from highly incomplete frequency information," IEEE Trans. Inf. Theory, vol. 52, no. 2, pp. 489-509, Feb. 2006.

[25] J. I. Peltoniemi, M. Gritsevich, and E. Puttonen, "Reflectance and polarization characteristics of various vegetation types," in Light Scattering Reviews. Berlin, Germany: Springer, 2015, pp. 257-294.

[26] J. Peltoniemi, T. Hakala, J. Suomalainen, and E. Puttonen, "Polarised bidirectional reflectance factor measurements from soil, stones, and snow," J. Quant. Spectrosc. Radiat. Transf., vol. 110, no. 17, pp. 1940-1953, Nov. 2009.

[27] J. I. Peltoniemi et al., "Reflectance of various snow types: Measurements, modeling, and potential for snow melt monitoring," in Light Scattering Reviews. Berlin, Germany: Springer, 2010, pp. 393-449.

[28] M. Gritsevich, T. Hakala, J. Peltoniemi, M. Paton, J. Stenman, and A. Luttinen, "Bidirectional reflectance measurements of meteorites acquired by FGI's field goniospectrometer," in Proc. Int. Meteor Conf., La Palma, Spain, 2013, pp. 205-209.

[29] O. Wilkman, M. Gritsevich, N. Zubko, J. I. Peltoniemi, and K. Muinonen, "Photometric modelling for laboratory measurements of dark volcanic sand," J. Quant. Spectrosc. Radiat. Transf., vol. 185 pp. 37-47, Dec. 2016.

[30] A. Kuusk, J. Kuusk, M. Lang, J. Peltoniemi, M. Gritsevich, and J. Pisek, "Reflectance reference target at Järvselja, Estonia for the calibration of optical remote sensing sensors and lessons learned," Int. J. Appl. Earth Observ. Geoinf., vol. 73, pp. 191-196, 2018. 